\title{
Die Euroskeptizismus-Spirale: EU-Berichterstattung und Medien-Negativität
}

\author{
Charlotte Galpin · Hans-Jörg Trenz
}

(C) Der/die Autor(en) 2018.

Zusammenfassung In der Euroskeptizismus-Forschung ist bislang der Frage nach den öffentlichen und medialen Vermittlungsprozessen, über die sich europakritische Meinungen formieren und Proteste gegen die EU ausweiten, nur wenig Beachtung geschenkt worden. In diesem Artikel soll die sich wandelnde Rolle der Nachrichtenmedien als Generator demokratischer Legitimität exemplarisch am Beispiel der Nachrichtenberichterstattung zur Wahl des Europäischen Parlaments 2014 nachgezeichnet werden. Im Ländervergleich zwischen Deutschland und Großbritannien wird untersucht, inwiefern die Europaberichterstattung durch einen systematischen Negativitätsbias geprägt wurde. Negativität als Nachrichtenwert fokussiert auf die Fehlleistungen der Politik, Polemiken, Skandale oder Krise. Damit, so wird unterstellt, untergraben die Medien das Vertrauen in die demokratische Politik und ihre Repräsentanten. Die Wirkung von Negativität kann in der Selektion, Rahmung, Verbreitung und Rezeption von EU-Nachrichten nachgewiesen werden. Auf der Grundlage standardisierter Inhalts- und Frameanalyse auf Artikel-Ebene, SprecherEbene und Rezipienten-Ebene kann der Negativitäts-Bias von EU-Nachrichten unterschiedlichen Amplifikatoren (Journalisten, politischen Akteuren und Nachrichtenkonsumenten) zugeordnet und zur Legitimität der Europapolitik im Kontext von demokratischen Wahlen in Bezug gesetzt werden. Von einer Euroskeptizismus-Spirale soll in dem Sinne gesprochen werden, dass sich im Laufe der medialen Kampagne Nachrichtenangebot und Nachfrage an Negativitätskriterien anpassen und gegenseitig in der Delegitimierung des europäischen Integrationsprojekts verstärken.

\section{Galpin $(\bowtie)$}

Department of Political Science and International Studies, Muirhead Tower, University of Birmingham, Birmingham, B15 2TT, Großbritannien

E-Mail: c.a.galpin@bham.ac.uk

\section{H.-J. Trenz}

Centre for Modern European Studies and Dept. of Media, Cognition, and Communication, Uni. of Copenhagen, Karen Blixens Vej 4, 2300 Kopenhagen, Dänemark

E-Mail: trenz@hum.ku.dk 
Schlüsselwörter Euroskeptizismus · Europäisches Parlament - Spitzenkandidaten · Europaberichterstattung · Framing $\cdot$ Mediennegativität $\cdot$ Medialisierung

\title{
The Spiral of Euroscepticism: EU News and Media Negativity
}

\begin{abstract}
The role of public and media processes of mediation in shaping Eurosceptic attitudes and driving opposition to the EU has received relatively little attention in Euroscepticism research. In this article, we examine media coverage of the 2014 European Parliament election to analyse the role of the media in generating democratic legitimacy. By comparing Germany and the UK, we investigate the extent to which news coverage of the EU suffers from a systematic negativity bias. Negativity as a news value focuses on political failures, polemics, scandals or crisis which can undermine trust in democratic politics and its representatives. Using standardised content and frame analysis of articles, actor statements and user comments, we demonstrate how a negativity bias in EU news can be amplified by different actors-journalists, political actors, and news readers - and linked to the legitimacy of EU politics in the context of democratic elections. We argue that we can speak of a 'spiral of Euroscepticism' that results from a negativity bias in media coverage and reception that that then contributes to delegitimising the European integration project.
\end{abstract}

Keywords Euroscepticism · European Parliament - Spitzenkandidaten · EU news · Framing $\cdot$ Media negativity

\section{Einleitung}

Der Euroskeptizismus ist Gegenstand der Parteienforschung und der politischen Meinungsforschung. Im Fokus stehen entweder die Produzenten des euroskeptischen Diskurses, also die Parteien, welche die Legitimität der Europäischen Union grundsätzlich herausfordern oder deren Rezipienten, also die Bürgerinnen und Bürger, die den Institutionen der EU ihre Unterstützung entziehen. In der Konzeptionalisierung des Phänomens geht es dabei oftmals um die Substanz, etwa im Sinne der Unterscheidung eines ,harten' und ,weichen' Euroskeptizismus (Taggart und Szczerbiak 2004) oder einer Positionierung von Parteien entlang einer pro- und antieuropäischen Spaltungslinie (Hooghe 2007). Aus soziologischer Perspektive ist vor allem aber auch die Frage nach den öffentlichen und medialen Vermittlungsprozessen von Relevanz, welche die Proteste gegen Europa kanalisieren, die Bürgerinnen und Bürger informieren und ihre Meinung zur Legitimität der EU bilden. Der Euroskeptizismus manifestiert sich entsprechend als eine Form der öffentlich-medialen Auseinandersetzung, welche die Legitimität der EU oder des europäischen Integrationsprozesses prinzipiell in Frage stellt und dabei einseitig negative und grundlegend ablehnende Einstellungsmuster gegenüber der EU und ihrer Protagonisten zum Ausdruck bringt (De Wilde und Trenz 2012). In der Dynamik dieser öffentlich-medialen Auseinandersetzungen spielt die Eigenlogik von Nachrichtenmedien eine entscheidende Rolle, die nicht nur als Amplifikatoren parteipolitischer Positionen oder als 
Spiegel der Meinungen der Bürgerinnen und Bürger in Erscheinung treten, sondern über selektive Nachrichtenrahmung in entscheidendem Maße zur Generierung demokratischer Legitimität der EU beitragen. In diesem Artikel soll die mediale Eigenlogik in der Selektion und Rahmung europapolitischer Nachrichten exemplarisch am Beispiel der Berichterstattung zur Wahl des Europäischen Parlaments 2014 nachgezeichnet werden. Im Ländervergleich zwischen Deutschland und Großbritannien wird untersucht, inwiefern die Europaberichterstattung durch einen systematischen Negativitätsbias geprägt wurde. Negativität als Nachrichtenwert fokussiert auf die Fehlleistungen der Politik, Polemiken, Skandale oder Krisen. Damit, so wird unterstellt, untergraben die Medien das Vertrauen in die demokratische Politik und ihre Repräsentanten (Kepplinger 1998; Soroka 2014).

Im Kontext der Wahlen zum Europäischen Parlament im Jahre 2014 konnten euroskeptische Parteien länderübergreifend Erfolge verbuchen. Die Negativität des öffentlichen Meinungsbildes zu Europa wird dabei zumeist auf die Mobilisierungsstrategien euroskeptischer Parteien zurückgeführt, die im Europa der Krise an Zulauf gewinnen (Nielsen und Franklin 2016). Allerdings unterscheiden sich europapolitische Debatten traditionell in der Themensetzung und der Dynamik diskursiver Auseinandersetzungen nach dem Muster segmentierter nationaler Öffentlichkeiten. Unterschiede in der Europäisierung des öffentlichen Meinungsbildes können dabei auf die Ausprägung nationaler Diskurskulturen zurückgeführt werden, die als ,soziokultureller Unterbau' für die länderspezifische Thematisierung Europas verantwortlich zeichnen (Hepp et al. 2012; Machill et al. 2006). Der Wahlerfolg euroskeptischer Parteien steht somit in einem ungeklärten Wirkungszusammenhang zur europapolitischen Medienberichterstattung und der medial zur Anwendung gelangenden Selektionsmechanismen und Interpretationsraster, durch die Themen einseitig sichtbar gemacht und Meinungen den Wählern vermittelt werden. Im Ländervergleich zwischen Deutschland und Großritannien lassen sich diese Unterschiede nationaler Diskurskulturen systematisch herausarbeiten und in einen Zusammenhang zur selektiven Wahrnehmung euroskeptischer Themen und Akteure stellen. Die europapolitische Debatte in Deutschland ist dabei traditionell von einem breiten, parteiübergreifenden Konsens mit Europa getragen, der von einem europafreundlichen Journalismus gestützt wird. In Großbritannien überwiegt demgegenüber ein konfrontativer Stil politischer Parteien sowie des Journalismus, die sich mit Europa kritisch-distanziert auseinandersetzen und europapolitische Entscheidungen aus einer Perspektive des nationalen Interesses in Frage stellen (Diéz Medrano 2003). Vor diesem Hintergrund sollen im Ländervergleich zwischen Deutschland und Großritannien nicht nur Unterschiede in der Parteienmobilisierung, sondern insbesondere auch journalistische Traditionen und mediale Eigenlogiken herausgestellt werden, die sowohl für stilistische Gemeinsamkeiten in der europapolitischen Nachrichtenberichterstattung als auch für national-spezifische Ausprägungen verantwortlich zeichnen.

Akteurs-zentrierte Ansätze in der Europaforschung analysieren die Verbreitung euroskeptischer Meinungsmuster im Zusammenhang mit Strategien des medialen Agenda-Settings, die von euroskeptischen Parteien erfolgreich umgesetzt werden. Mediennegativität wird als Derivat der Mobilisierungsstrategien politischer Parteien oder euroskeptsicher Bewegungen angesehen. So wird z. B. das Charisma oder die Geschicklichkeit der Führer euroskeptischer Bewegungen hervorgehoben, die Mas- 
senmedien für ihre Zwecke der Anti-EU Propaganda einzuspannen (Adam 2009; de Vries und Edwards 2009). Auch die Defizite der Öffentlichkeitsarbeit der europäischen Kommission werden zuweilen für die verzerrte Darstellung der Europapolitik in den nationalen Medien zur Verantwortung gezogen (Brüggemann 2010; van Brussel 2014). Aufgrund dieser akteurs-zentrierten Fokussierung in der Forschung ist den intermediären Prozessen der Kommunikation, Interpretation und Rahmung von Wissensvorräten und Meinungen zur EU nur wenig Beachtung geschenkt worden. Zur Öffnung dieser Black-Box der medialen Vermittlung muss der Euroskeptizismus im Zusammenhang mit den Produktionsbedingungen von Nachrichten und den journalistischen Praktiken der Nachrichtenselektion und Rahmung diskutiert werden.

Statt den Euroskeptizismus in den Medien auf exogene, akteurs-spezifische Erklärungsfaktoren zurückzuführen, soll in diesem Beitrag ein endogener, institutionentheoretischer Ansatz gewählt werden, der die sich wandelnde Rolle der Nachrichtenmedien als Generator demokratischer Legitimität nachzeichnet und daran anschließend am Beispiel der Europaberichterstattung den Entstehungs- und Verbreitungskontext von EU-Nachrichten eine vergleichenden Analyse unterzieht. Unsere These ist, dass die Nachrichten zur Europawahl in beiden Ländern durch einen systematischen Negativitätsbias der Berichterstattung geprägt wurden (Abschn. 2). In einer ersten Annäherung soll hierzu die Eigenlogik der Massenmedien in der Produktion und Amplifikation von EU-Nachrichten nachgezeichnet werden (Abschn. 3). Darauf aufbauend können die Spezifika von Negativität als Nachrichtenwert herausgestellt und operationalisiert werden (Abschn. 4-6). Die Wirkung von Negativität kann dann in der Selektion, Rahmung, Verbreitung und Rezeption von EU Nachrichten nachgewiesen werden. Auf der Grundlage standardisierter Inhalts- und Frameanalyse auf Artikel-Ebene, Sprecher-Ebene und Rezipienten-Ebene kann der NegativitätsBias von EU-Nachrichten unterschiedlichen Amplifikatoren (Journalistinnen und Journalisten, politische Akteure und Nachrichtenkonsumenten) zugeordnet und zur Legitimität der Europapolitik im Kontext von demokratischen Wahlen in Beziehung gesetzt werden (Abschn. 7). Dabei ist auf die segmentierte Ausprägung des Negativitätsbiases in Anpassung an nationale Diskurskulturen hinzuweisen mit dem Befund einer genuin euroskeptischen und selbstbezogenen Debatte in Großritannien und eines importierten Euroskzeptizismus in der medialen Debatte in Deutschland, die einen gesamteuropäischen Beobachtungsraums als Bezugspunkt für die Wahlberichterstattung einnimmt. Unsere abschließenden Betrachtungen beziehen sich auf die Auswirkungen von Negativität als Nachrichtenwert auf die demokratische Politik der EU. Von einer Euroskeptizismus-Spirale soll in dem Sinne gesprochen werden, dass sich im Laufe der medialen Kampagne das Angebot und die Nachfrage von Nachrichten an Negativitätskriterien anpassen und gegenseitig in der Delegitimierung des europäischen Integrationsprojekts verstärken (Abschn. 8).

\section{Der Euroskeptizismus aus institutionstheoretischer Perspektive}

Europapolitische Richtungsentscheidungen werden zunehmend zur Zielscheibe parteipolitischer Auseinandersetzungen, die sich nicht an bekannten ideologischen Mustern orientieren, sondern entlang einer neuen identitären Spaltungslinie zwischen 
EU-Befürwortern und Gegnern ausrichten (Hooghe und Marks 2009). Von den EUBefürwortern wird der fortschreitenden Vergemeinschaftung Vertrauen geschenkt. Soziodemographisch lässt sich diese Gruppe zumeist als Integrationsgewinner verorten, die über ein hohes transnationales Kapital im Sinne von Bildung und Sprachkompetenzen verfügen (Gerhards et al. 2016). EU-Gegner hingegen finden sich häufig auf der Verliererseite der Europäisierung und Transnationalisierung. Viele fühlen sich von national-populistischen Bewegungen angezogen und möchten den Integrationsprozess zurückfahren (Kriesi et al. 2012; Teney et al. 2013; für Deutschland siehe auch Kiess et al. 2017). In diesem Beitrag wenden wir uns dem Euroskeptizismus aus institutionstheoretischer Perspektive zu und stellen die Frage nach dem Feld symbolischer Auseinandersetzungen, welche eine Fundamentalopposition gegen die EU antreiben. Der Euroskeptizismus kann als eine Folge der supranationalen Herrschaftsausübung interpretiert werden, welche sich bestimmten Mechanismen der politischen Legitimation unterwerfen muss. Demokratische Legitimität wird dabei zum Orientierungshorizont für die Beurteilungen der Bürgerinnen und Bürger, wenn ihre Mitentscheidung beispielsweise bei Wahlen gefragt ist (Bach 2008). Europäische Institutionen und Entscheidungsakteure können aber nicht nur auf die passive Zustimmung der Bürgerinnen und Bürger setzen (der alte sogenannte permissive Konsens der EU), sondern müssen sich auch vielfach mit Partizipationsansprüchen und gesellschaftlichen Widerständen auseinandersetzen. Der Euroskeptizismus entfaltet eine Form von delegitimierender Öffentlichkeit. Herrschaftssoziologisch betrachtet handelt es sich dabei aber nicht nur um eine Spielart der systeminternen Kritik, etwa in der Art eines Rufes nach Rechtfertigung und verbesserter politischer Teilhabe. Die euroskeptische Opposition ist vielmehr grundsätzlicher Art, indem sie die Systemlegitimation der EU, also ihre fundamentalen Werte und Grundsätze, ihre Autorität als supranationale Entscheidungsinstanz und ihre Weichenstellungen für die Zukunft in Frage stellt (de Wilde und Trenz 2012). Die Euroskeptiker kontern den breiten Elitenkonsens etablierter Parteien und ihre Unterstützung des Integrationsprozesses mit ihrem Ruf nach Renationalisierung. Dieser Agenda wird in allen europäischen Ländern mit wechselndem Erfolg von euroskeptischen Oppositionsparteien am linken oder am rechten Rand des politischen Spektrums besetzt, kann in einzelnen Fällen aber auch von Parteien der politischen Mitte (etwa die britische Conservative Party) oder anderen hohen Repräsentanten (etwa der ehemalige tschechische Staatspräsident Klaus) aufgegriffen werden. Euroskeptiker allen Couleurs bedienen sich dabei oftmals populistischer Mobilisierungsstrategien, also einer Form der symbolischen Macht, die sich nicht im Repräsentationsgefüge parlamentarischer oder parteipolitischer Auseinandersetzungen ausbildet, sondern sich auf die Stimme des Volkes als eine Art unvermittelte Legitimationsressource beruft (Arditi 2007).

Im Wahlkampf kann sich der Euroskeptizismus allerdings nicht allein auf das Charisma seiner Protagonisten verlassen. Als eine Form von delegitimierender Öffentlichkeit unterliegen die den Euroskeptizismus antreibenden symbolischen Auseinandersetzungen vielmehr bestimmten Regeln des politischen Diskurses. Hierzu zählen insbesondere die normative Begründbarkeit und kommunikative Vermittelbarkeit ihrer Positionen, die nicht nur an die politischen Kontrahenten gerichtet sind, sondern vor allem darauf abzielen, ein diffuses Publikum zu überzeugen und 
emotional aufzuwühlen. Wie in diesem Beitrag nachgezeichnet werden soll, werden diese in der Form einer Fundamentalopposition zur EU ausgetragenen Legitimitätskämpfe in entscheidendem Maße durch mediale Infrastrukturen und Logiken massenmedialer Kommunikation geprägt. Dabei geht es nicht allein darum, dass sich der Kampagnenstil populistischer Parteien erfolgreich an mediale Aufmerksamkeitskriterien anpasst. In einem viel fundamentaleren Sinne soll vielmehr die institutionelle Eigenlogik politischer Nachrichtenmedien, und hierbei insbesondere die Selektionsregeln und kulturellen Rahmungsmuster der Europaberichterstattung, herausgearbeitet und im Zusammenhang mit der Verbreitung euroskeptischer Diskurs- und Einstellungsmuster diskutiert werden. Um diese institutionelle Eigenlogik der Massenmedien erfassen zu können, darf nicht nur die neutrale Vermittlungsfunktion in der Verbreitung europapolitischer Nachrichten im Vordergrund stehen, die es europaskeptischen Parteien ermöglicht, mit ihren Wählern in Kontakt zu treten. Für das Verständnis der Verbreitung euroskeptischer Meinungsbilder ist darüber hinaus ein systematischer Negativitätsbias in der Beurteilung von EU Politik von Relevanz, der sich auf die Nachrichtenselektion und Rahmung auch ohne die gezielte Intervention euroskeptischer Akteure auswirkt.

\section{Zur institutionellen Logik der Massenmedien}

Von einer Logik der Massenmedien soll hier in einem nicht-deterministischem Sinne gesprochen werden, also im Unterschied zur Medienwirkungsforschung, die primär am Nachweis direkter Auswirkungen des mediales Diskurses auf die Meinungen und Einstellungsmuster des Publikums interessiert ist. Der Begriff ,Medienlogik ist von Altheide und Snow eingeführt worden als Sammelbegriff für die formalen und informellen Regeln und Codes, welche die Produktionsroutinen medialer Inhalte anleiten (Altheide und Snow 1979). Statt direkter kausaler Zusammenhänge sollen institutionelle Anpassungsprozesse beschrieben werden, also die Frage, wie sich politische Kommunikationslogiken und mediale Logiken angleichen, beispielsweise dadurch, dass euroskeptische Parteien erfolgreiche Strategien zur Erzeugung medialer Aufmerksamkeit voneinander kopieren oder dass sie einheitliche Nachrichtenwerte in der Selektion und Rahmung von Inhalten zugrunde legen, um damit den Erwartungshaltungen ihres Publikums zu entsprechen. ${ }^{1}$ Hierzu zählt insbesondere auch die Frage, wie Medien die Institutionalisierung demokratischer Prozesse anleiten und zu derer Legitimierung beitragen. Einige Autoren sprechen diesbezüglich auch von einer Medialisierung demokratischer Entscheidungsprozesse, wonach die Medienlogiken zur Erzeugung gesellschaftlicher Aufmerksamkeit zunehmend die Funktionsweise des demokratischen Prozesses und seiner Institutionen (beispielsweise politische Parteien) mitbestimmen (Hjarvard 2013; Marcinkowski 2005; Marcinkowski und Steiner 2009). Medien, und speziell der Journalismus, behaupten als eigenständige Institution ihre Unabhängigkeit, etwa gegenüber der Politik und bilden entsprechende Kapazitäten aus (materielle und symbolische Ressourcen, professio-

\footnotetext{
1 Im Neo-Institutionalismus sind solche Prozesse auch also Isomorphismus bezeichnet worden (DiMaggio and Powell 1983).
} 
nelle Routinen, formelle und informelle Regeln), die es ihnen erlauben, den Prozess der permanenten Nachrichtenproduktion in Gang zu setzen. Zugleich besetzen die Medien aber auch eine prominente Rolle als Hersteller gesellschaftlicher Sichtbarkeit und damit als Konstrukteur gesellschaftlicher Wirklichkeit (Marcinkowski und Steiner 2009; Nassehi 2002). Aus letzter Perspektive werden auch politische Institutionen oder wissenschaftliche Institutionen einer Medienlogik unterworfen, d.h. sie werden ,medialisiert', indem sie sich an die technologischen, wirtschaftlichen oder auch ästhetischen Entscheidungs- und Selektionsprozesse zur Produktion medialer Inhalte anpassen.

Unter Hinweis auf solche endogenen Medienlogiken kann die journalistische Praxis in einem Feld verortet werden, das durch die Kräfteverhältnisse verschiedener, im Konkurrenzkampf zueinander stehender journalistischer Unternehmen gekennzeichnet ist (Bourdieu 1998; Hanitzsch 2016). In seinem Essay über das Fernsehen beschreibt Pierre Bourdieu die Produktionsbedingungen des journalistischen Feldes. Der Kampf um Meinungen ist im Wesentlichen ein Kampf um Marktanteile. Auf oberer Ebene kommt es zur Ausbildung von marktbeherrschenden Monopolen, wie etwa die Murdoch Presse in Großbritannien, die in der europapolitischen Auseinandersetzung eine dezidiert anti-europäische Position einnimmt und gegen Marktkonkurrenten erfolgreich verteidigt. ${ }^{2}$ Auf unterer Ebene wird der Meinungskampf von den Journalisten selber ausgetragen, die sich ständig auf der Jagd nach dem Scoop (der Exklusivmeldung) befinden. Diese Jagd ist allerdings kein rein wirtschaftliches Kalkül, obwohl sie in ökonomischen Faktoren begründet liegt. Nachrichtenselektion wird auch zum Kulturkampf, in dem sich Journalisten beispielsweise als Verteidiger der Demokratie oder der Integrität des Nationalstaats in Szene setzten. Die Aufgabe der Journalistinnen und Journalisten in der Nachrichtenselektion und Rahmung verlagert sich damit von der neutralen Vermittlung von Politik zu ihrer Inszenierung und Dramatisierung. Journalistinnen und Journalisten müssen sich sozusagen selber als Stimme in den symbolischen Auseinandersetzungen um Legitimität behaupten und beispielsweise ihren Status gegen die Politik durchsetzen. Nachrichtenwerte dienen dabei der Autonomiesicherung gegenüber der Politik, indem sie das politische Geschehen rekontextualisieren und in ökonomische und Statusgewinne für die Journalistinnen und Journalisten überführen. Bourdieu bezeichnet diese Eigenlogik der Medien in der Nachrichtenselektion als unsichtbare Zensur: Sowohl das Was (die Themen) als auch das Wie der Präsentation dieser Themen sind durch Medienformate vorgegeben, wie Bourdieu am Beispiel des Aufruhrs moslemischer Jugendliche in den Banlieus von Paris vorführt, wo es den Journalistinnen und Journalisten vornehmlich darum geht, das Ungewöhnliche und Außerordentliche darzustellen und eine Polemik gegen bestimmte Akteursgruppen (Ausländer) voranzutreiben, statt Kritik an den sozialen Verhältnissen zu üben.

\footnotetext{
2 Oliver Daddow (2012) beschreibt die euroskeptische Wende der britischen Presse als ,Murdoch Effekt”. Euroskeptizismus ist dann nicht nur ,big business", sonder auch eine gezielte Form der politischen Einflussnahme (Robert Murdoch wird oftmals vorgeworfen, dass er deswegen anti-europäisch eingestellt sei, weil man ihn in Brüssel einfach ignoriere, wohingegen ihm in Downing Street die Türen offen stehen).
} 
In Anlehnung an Bourdieu kann auch die Arbeit der EU-Korrespondenten innerhalb des professionellen Feldes des Journalismus verortet werden (Hummel 2006). Von Interesse ist etwa, wie sich ein bestimmter Habitus Brüsseler Korrespondenten herausbildet, die sich als kritische Stimme Europas in Szene setzen und ein Monopol in der Interpretation der Gemeinschaftspolitik beanspruchen. In der Europaberichterstattung kommt es dabei häufig zur Konfrontation journalistischer Standards, wobei sich der Marktwert europäisch-gerahmter Nachrichten nur schwer durchsetzt. Auch in der Europaberichterstattung kommen deshalb Schablonen für die schnelle Herstellung standardisierter Nachrichten zum Einsatz, wobei ein euroskeptischer Blick vorherrscht, der durch Polemik gegen EU-Institutionen getrieben ist und die EU vornehmlich durch die Brille der nationalen Politik wahrnimmt. Im Sinne eines ,diskursiven Institutionalismus ' ist die Nachrichtenberichterstattung weitestgehend auf die Schaffung von Resonanz in nationalen Diskursräumen ausgerichtet, wobei sich Nachrichtenwerte und national spezifische Diskurstraditionen aufeinander einspielen (Hepp et al. 2012; Schmidt 2008). Journalistinnen und Journalisten lernen also, sich etwa auf euroskeptische Erwartungshaltungen ihrer Leserinnen und Leser einzustellen, um diese dann gleichermaßen durch die negative Tonalität der Berichterstattung zu verstärken.

Unter Annahme einer solchen medialen Eigenlogik der Nachrichtenselektion und Rahmung kann der Einfluss der Nachrichtenmedien auf den demokratischen Prozess der EU einer systematischen Untersuchung unterzogen werden. Damit öffnet sich eine neue Perspektive auf den Euroskeptizismus nicht als unabhängiger parteipolitischer Mobilisierungsfaktor oder als Antriebskraft demokratischer Delegitimierung, sondern als Nebenprodukt medialer Vermittlung. Statt den direkten Einfluss euroskeptischer Mobilisierung auf die Wählerentscheidungen zu messen, beobachten wir, wie euroskeptische Akteure, Positionen und Diskursinhalte in der Vermittlung europäischer demokratischer Prozesse (die Berichterstattung zur Europawahl 2014) selektiv aufgegriffen und gerahmt werden. Der relative Erfolg euroskeptischer Kampagnen als Ergebnis der Wahlen wird somit nicht als unmittelbares Resultat parteipolitischer Mobilisierung interpretiert, sondern als Ergebnis einer erfolgreichen institutionellen Rückkoppelung zwischen Mobilisierungslogiken politischer Parteien, Medienlogiken und entsprechenden Erwartungshaltungen des Publikums. Ein solcher Erfolg stellt sich dadurch ein, dass politische Botschaften mit Nachrichtenwerten koordiniert werden und entsprechende Selektionsmechanismen in der Vermittlung europäischer Politik zur Anwendung gelangen, die wiederum das Publikum aktivieren, der europäischen Politik selektive Aufmerksamkeit zu schenken. Euroskeptische politische Parteien und Medieninstitutionen sind dann synchronisiert, in dem Sinne, dass ihre Wählerschaft und Leserschaft über ähnliche Codes zu erreichen sind. Um welche Codes der selektiven Nachrichtenproduktion es sich dabei im Einzelnen handelt, kann mit Hinweis auf die Nachrichtenwerttheorie abgehandelt werden. 


\section{EU-Berichterstattung und Mediennegativität}

Nachrichtenselektion ist werteorientiert. In der Tradition von Galtung und Ruge (1965) haben Medienforscher eine lange Liste von Nachrichtenwerten vorgelegt, nach denen sich Journalisten in der Selektion und Rahmung politischer Nachrichten ausrichten. Ein konsistenter Befund der Nachrichtenwerte Forschung ist, dass schlechte Nachrichten den guten Nachrichten vorgezogen werden. In Zeitreihenuntersuchungen konnte dabei in unterschiedlichen westlichen Demokratien eine generelle Zunahme der Nachrichtennegativität seit etwa den frühen Siebziger Jahren konstatiert werden, die mit einem wachsenden kritischen Bewusstsein der Leser, aber auch mit dem Verlust an Vertrauen in Demokratie in Zusammenhang gebracht wird (Cappella und Jamieson 1997; Kepplinger 1998; Patterson 2000). Der systematische Negativitätsbias in der politischen Nachrichtenberichterstattung weist in diesem Sine auf eine sich wandelnde Rolle der Nachrichtenmedien in der Generierung demokratischer Legitimität. Statt ausgewogener Berichterstattung oder begründeter Kritik bedienen sich Journalisten zunehmend polemischer und exzessiver Stilmittel und zeichnen ein negatives Zerrbild politischer Prozesse. Dies setze eine ,Spirale des Zynismus' in Gang, die das Vertrauen in demokratische Politik unterminiert (Cappella und Jamieson 1997).

Mediennegativität korreliert des Weiteren mit der Distanz der Ereignisse, über die berichtet wird. Nähe scheint demgegenüber eher ein Bedürfnis für positive Nachrichten zu wecken. Der Mediennegativitätsbias wirkt von daher insbesondere in der Auslandsberichterstattung, wo andere Nachrichtenwerte, wie Familiarität, Personalisierung oder kulturelle Nähe als Stilmittel nur bedingt zur Verfügung stehen (de Vreese und Kandyla 2009; Entman 2004). Die Aufmerksamkeit für ferne Ereignisse jenseits des vertrauten Horizonts nationaler Politik kann leichter geweckt werden, wenn durch sie Drama und Konflikt assoziiert werden, wenn die Integrität bestimmter Akteure oder Institutionen untergraben wird, oder wenn Nachrichten Gefühle des Zweifels, der Unsicherheit oder Angst hervorrufen. Auch in der EUNachrichtenberichterstattung ist vielfach beklagt worden, dass Nachrichtennegativität die Vertrauensbildung der Bürgerinnen und Bürger unterminiert (De Vreese 2003; Trenz 2005a, 2008). In den Nachrichtenmedien wird die EU-Politik bevorzugt mit Misswirtschaft und Skandalen in Verbindung gebracht. Statt die Konsensfähigkeit der EU hervorzuheben, fokussieren die Journalistinnen und Journalisten lieber auf Streit; statt die erhöhte Problemlösungsfähigkeit europäischer Regelungen zu loben, beklagen sie Überregulierung und Krise; und statt die komplexe Legitimität der EU im Mehrebenengefüge darzustellen, reduzieren die Medien Legitimität auf einfache Ja-Nein Muster in der quasi-intuitiven Zustimmung oder Ablehnung europäischer Autorität.

In der nationalen Nachrichtenberichterstattung wird der Negativitätsbias zudem durch das Objektivitätsgebot ausgewogen, demzufolge von Journalistinnen und Journalisten erwartet wird, sich dem innenpolitischen Geschehen unparteiisch 
zuzuwenden. ${ }^{3}$ Auch wenn gerade Journalistinnen und Journalisten der Boulevardpresse oftmals die ethischen Grundsätze der Fairness übertreten, so können tendenziöse Berichterstattung und verzerrte Nachrichten doch leichter identifiziert und auf zugrundeliegende Nachrichtenwerte wie Sensationalismus, Singularität, oder Verbrechen rückgeführt und, wenn notwendig, korrigiert werden. Ein solche Schablone zur Objektivitätsmessung lässt sich auf die EU-Berichterstattung nur bedingt anwenden. Von Journalistinnen und Journalisten wird vielmehr erwartet, dass sie die nationalen Interessen herausstellen, um die EU-Politik für ihre Leserinnen und Leser relevanter zu machen (Örnebring 2013). Das Gebot der Ausgewogenheit und Fairness gilt dabei nur eingeschränkt. Im Gegenteil sollen die nach Brüssel gesandten Korrespondenten die Fülle von EU Nachrichten nach nationalen Relevanzkriterien filtern und beispielsweise in der Wahlkampfberichterstattung zum Europäischen Parlament die Kandidaten des eigenen Landes mit Vorzug behandeln. Diese eingeübten Modelle eines journalistischen Nationalismus sind fester Bestandteil journalistischer Kulturen und Sozialisationsmuster (Hannerz 2004). Eine weitere in diesem Zusammenhang wichtige Einschränkung des Objektivitätsprinzips von Nachrichten lässt sich auf die Eigenart von EU Debatten zurückführen, oftmals die Frage nach der Legitimität der EU und des europäischen Integrationsprozesses auf grundsätzliche Art und Weise aufzuwerfen. Journalistinnen und Journalisten stellen mit Blick auf die EU gerne die Frage nach der ,Polity ' oder ,System“ Legitimation. Dies erlaubt ihnen eine Dramatisierung des politischen Geschehens, aber auch eine eigene Positionierung, indem sie sich selber als Befürworter oder Gegner des Integrationsprozesses in Szene setzen. Diese Art und Weise der ,Polityoder Systemrahmung' kommt aber auch den Euroskeptikern entgegen, die genau solche Debatten nutzen, um von dem tagespolitischen Geschäft des europäischen Regierens abzulenken und einen generellen Zweifel an der Legitimität der EU zu wecken (de Wilde et al. 2013). Von Journalistinnen und Journalisten wird dann zwar einerseits erwartet, sich von den ,extremistischen' Positionen der Parteien am Rande des politischen Spektrums abzugrenzen. Gleichzeitig greifen sie die von euroskeptischen Parteien propagierte Negativität als Nachrichtenwert auf und passen sich deren Agenda der Fundamentalkritik der EU an.

In der Medienwirkungsforschung wird auf einen Zusammenhang zwischen Negativität von Nachrichten und der Delegitimierung des repräsentativ-demokratischen Systems von Politik hingewiesen. Dies misst sich beispielsweise im wachsenden Zynismus und Vertrauensverlust der Wähler in demokratische Institutionen oder auch generell in einem Rückgang der Beteiligung bei demokratischen Wahlen. In der Europaberichterstattung konnte ein Kausalitätszusammenhang zwischen Negativität von Nachrichten und Zynismus der Wählermeinungen experimentell durch Studien mit Nachrichtenkonsumenten nachgewiesen werden: Wer mit negativen EU-Nachrichten konfrontiert wird, neigt zu euroskeptischen Einstellungsmustern, schenkt den

\footnotetext{
3 Wir lassen hierbei die Frage eines grundsätzlichen Wertewandels und einer Reorientierung des Journalismus in Anpassung an kommerzielle Marktlogiken offen, konzedieren aber, dass das journalistische Objektivitätsgebot vor allem in Teilen der britischen Presse als Richtschnur journalistischer Arbeit zunehmend in Frage gestellt und von der Boulevardpresse durch gezielte politische Einflussnahme konterkariert wird.
} 
EU-Institutionen weniger Vertrauen und ist auch nur noch bedingt bereit, sich an den europäischen Parlamentswahlen zu beteiligen (de Vreese 2007).

\section{Die Wahlen zum Europäischen Parlament: Von ,second order' zu ,first order'?}

Um diesen Zusammenhang zwischen medialer Vermittlungslogik und Euroseskeptizismus weiter zu beleuchten, soll im Folgenden auf die allgemeinen Vermittlungsprobleme europäischer Politik und hierbei insbesondere die Probleme der Herstellung einer demokratisch-legitimierenden Öffentlichkeit näher eingegangen werden. Die Institutionalisierung demokratischer Entscheidungsverfahren der EU ist einerseits auf die Verfügbarkeit medialer Dienstleistungen angewiesen, trifft anderseits aber auch immer wieder auf Widerstände struktureller und ideeler Art in der Form eines nationalstaatlich verankerten und oftmals nationalistisch eingestellten Journalismus, der nur unzureichende Kapazitäten für die EU-Berichterstattung bereitstellt (Trenz 2005b). Das somit bezeichnete europäische Öffentlichkeitsdefizit stellt eine Strukturbarriere für die Demokratisierung der EU dar. In der Berichterstattung zur den Wahlen des Europäischen Parlaments wird beispielsweise vornehmlich aus nationaler Perspektive berichtet. Europäische Akteure und europäisch gerahmte politische Fragestellungen gelten als zweitrangig, womit eine informierte politische Willensbildung erschwert und der Wahlentscheid allenfalls als ,second order' gelten kann (Reif und Schmitt 1980; Hix und Marsh 2007; de Wilde et al. 2013).

Mit den Wahlen zum Europäischen Parlament im Jahre 2014 sollte diesen Tendenzen einer Renationalisierung und Fragmentierung des Wahlkampfes wirkungsvoll entgegengetreten werden. Als Innovation galten die sogenannten Spitzenkandidaten: die von den europäischen Parteienkoalitionen aufgestellt wurden, um für das Amt des EU-Kommissionspräsidenten zu kandidieren. Spitzenkandidaten sollten in gesamteuropäischen Wahlkämpfen auftreten und damit den Anspruch des Europäischen Parlaments auf genuine demokratische Legitimation bekräftigen. Damit würden sich europäische Parlamentswahlen als ,first order election “ durchsetzen und eine informierte Meinungs- und Willensbildung der gesamteuropäischen Bevölkerung ermöglichen (Gattermann et al. 2016; Schmitt 2005).

Die Spitzenkandidatenkampagne als eine Strategie des transnationalen medialen Agenda-Settings bietet sich somit als Testfall an, um die hier zugrundegelegte These einer institutionellen Eigenlogik der Nachrichtenmedien auszutesten. Gemä $\beta$ unserer Erwartungen werden solche exogenen Mobilisierungsstrategien durch mediale Eigenlogiken in der Selektion und Rahmung europapolitischer Nachrichten ausgebremst. Die über die Spitzenkandidatenkampagne in Szene gesetzten demokratischen Beteiligungsverfahren treten in die Euroskeptizismus-Falle. Wir erwarten:

1. eine Amplifikation von Mediennegativität in der Berichterstattung zur europäischen Parlamentswahl in

a) der journalistischen Nachrichtenselektion,

b) der Interpretation durch Sprecher in den Medien, und

c) der Kommentierung von Nachrichten durch die Rezipienten. 
Wir erwarten ferner:

2. eine Form der medialen Streitpolitik, die sich mit Grundsatzfragen der Legitimität des politischen Systems der EU auseinandersetzt, und der von den Spitzenkandidaten eingeleiteten Form der parteipolitischen Auseinandersetzung nur wenig Beachtung schenkt.

Damit werden:

3. euroskeptische Meinungsmuster und Akteure mit Aufmerksamkeit belohnt und in den Mittelpunkt von Kampagnen gestellt, welche die Meinungsbildung der Europäer vorantreiben.

Im Ländervergleich zwischen Deutschland und Großbritannien können damit:

4. Unterschiede in den journalistischen Traditionen und medialen Eigenlogiken herausgestellt werden, die für ein differenziertes Politisierungsmuster der EU mitverantwortlich zeichnen und damit entscheidend zur Dynamik der Spiztenkandidatenkampagne und der durch sie ausgelösten Legitimationseffekte beitragen.

\section{Zur Messung des Negativitätsbiases in der Nachrichtenberichterstattung}

Als empirische Grundlage der vorliegenden Untersuchung dient die Erhebung der Berichterstattung zur Europawahl auf den wichtigsten online Nachrichtenportalen in Großbritannien und Deutschland für einen Zeitraum von drei Wochen (zwei Wochen vor und eine Woche nach dem Wahltermin am 22-25. Mai 2014). ${ }^{4}$ Artikel (Nachrichtenartikel, Hintergrundartikel und Kommentare) werden über ein Set von Stichworten zum Thema ,Europawahlen“ aus dem online Archiv der Nachrichtenportale extrahiert. Um den journalistischen Nachrichteninput mit unterschiedlichen Formen der Rezeption von Lesern konfrontieren zu können, werden zusätzlich zu den Artikeln auch die online Leser-Kommentare erfasst, die nach Möglichkeit in chronologischer Ordnung abgespeichert werden, wobei sowohl Hauptkommentareinträge, als auch Antworten auf die Kommentare anderer Leserinnen und Leser in das Sample einfließen. Unsere Codierung basiert auf einem zufallsgenerierten, stratifizierten Sample von $50 \%$ aller Artikel. ${ }^{5} \mathrm{Zu}$ jedem dritten Artikel wurden bis zu 20 Kommentare in chronologischer Reihenfolge ihres Erscheinens kodiert. Ins-

\footnotetext{
${ }^{4}$ Das Sample umfasst für beide Länder zwei Qualitätszeitungen (links-liberal und konservativ) und einer Boulevardzeitung. Die online Nachrichtenportale (welt.de, spiegel.de und bild.de für Deutschland und guardian.co.uk, telegraph.co.uk and dailymail.co.uk für Großbritannien) wurden nach ihrem Ranking der Besucherstatistiken und nach ihrer Repräsentativität für die Qualitätspresse und den Boulevardjournalismus des jeweiligen Landes ausgewählt. Es sei darauf hingewiesen, dass die Online Nachrichtenberichterstattung dieser Portale nicht identisch ist mit den Druckausgaben der jeweiligen Zeitungen bzw. Nachrichtenmagazine.

5 Die Auswahl von 50\% aller Artikel bezieht sich auf die jeweiligen Zeitungen, nicht auf das Gesamtsample.
} 
gesamt gehen 335 Artikel, 1128 Sprecheraussagen und 2029 Kommentare in die Untersuchung ein.

Um dem Spektrum der von den Nachrichtenmedien angeleiteten Meinungsbildungsprozessen gerecht werden zu können, erfolgt die Messung von Mediennegativität auf drei verschränkten Ebenen:

a) die Ebene der journalistischen Nachrichtenselektion und des Framings (Nachrichten-Story Negativität, die dem Journalismus zugeordnet werden kann);

b) die Ebene politisch-medialer Auseinandersetzung (Quellen-Negativität der ebenfalls journalistisch vermittelten Auswahl von Sprechern in den Medien); und

c) die Ebene der Lesermeinung und Rezeption (Kommentar-Negativität, die den Nachrichtenrezipienten zugeordnet werden kann).

Codierungsanweisungen zur Messung von Negativität von Nachrichten sind der Studie von Lengauer et al. (2012) entnommen. Auf der generellen Nachrichtenebene und Sprecherebene erfolgt die Messung von Negativität durch die Erhebung sogenannter generic frames. Dabei handelt es sich nicht um qualitativ zu rekonstruierende ,interpretive frames' (Patterson 2000, S. 11), sondern um eine quantitative Messung der Tonalität von Nachrichten anhand binärer Codes. Solche ,generic frames ' eignen sich insbesondere für die ländervergleichende Analyse, da sie universell und nicht kulturspezifisch zur Anwendung gelangen und in der Regel auch durch die Codierer zuverlässig identifiziert werden können. Auf Nachrichten Ebene verorten wir die Tonalität der Story auf einer Skala von negativ ( -1$)$, neutral/ambivalent (0) bis positiv (1). Zusätzlich codieren wir die Zielscheibe von Nachrichten-Story Negativität. Wir unterscheiden zwischen spezifischer Negativität, die sich gegen bestimmte Akteure richtet (z. B. gegen die EU-Kommission oder gegen nationale populistische Parteien) und diffuser Negativität, die sich allgemein gegen Politik oder gegen das System wendet (gegen Europa, die EU oder Brüssel). Damit soll gemessen werden, ob Negativität von den Journalistinnen und Journalisten kanalisiert, und in Kritik gegen bestimmte Akteure (nationale oder europäische) übersetzt wird, oder ob sich Negativität als allgemeine Unzufriedenheit oder Wut gegen die EU Ausdruck verschafft. Tonalität wurde mit Hilfe eines Schlagwortkatalogs (z.B. Versagen, Krise, Frust, Wut versus Erfolg, Errungenschaft, Lösungen, Begeisterung, Hoffnung usw.) codiert.

Auf Sprecher-Ebene wenden wir uns den Inhalten von Sprechaussagen namentlich zitierter Akteure als Teil von Nachrichten-Stories zu. Dabei vernachlässigen wir die Selektionsleistung von Sprecheraussagen durch die Journalistinnen und Journalisten und bewerten den Inhalt, wie er medial wiedergegeben wird. Zur Messung von Sprechaussagen greifen wir auf das von Koopmans und Statham entwickelte Schema der claims-making Analyse zurück (Koopmans und Statham 2010). Wir codieren Akteurstypus (Regierung, Legislative, Partei, etc.), Reichweite (EU, national, regional), Länderzugehörigkeit sowie Parteizugehörigkeit. Über diese Variablen kann der Erfolg von Agenda-Setting Strategien der Spitzenkandidatenkampagne direkt gemessen und zum relativen Erfolg euroskeptischer Kampagnen in Beziehung gesetzt werden (Gattermann und Vasilopoulou 2017). In Anlehnung an die Messung von Mediennegativität auf Artikel Ebene messen wir spezifische und diffuse Tonalität von Akteursaussagen auf einer 3-Werte Skala (negativ, neutral und posi- 
tiv). Wir vermuten, dass euroskeptische Positionen insbesondere von Parteien am rechten Rand (die britische UKIP und die deutsche AfD) zum Ausdruck gebracht werden, wohingegen Mitte-rechts, Mitte-links, liberale und grüne Parteien die Spitzenkandidatenkampagne unterstützen sollten und ja auch mit eigenen Kandidaten ins Rennen getreten sind. Die deutsche Linke entzieht sich einer einfachen pround antieuropäischen Schematisierung. Eine Sonderrolle nimmt ferner die britische Conservative Party ein, von deren Fraktion im Europäischen Parlament kein eigener Kandidat für das Amt des Kommissionspräsidenten aufgestellt wurde. Neben dem Ländervergleich und dem Vergleich von Parteienpositionen werden auch Unterschiede zwischen Nachrichtenformaten (Qualitäts- und Boulevardpresse) sichtbar.

Die Codierung erfolgte durch vier Codierer auf der Basis eines standardisierten Codebuchs durch Eingabe der Daten in eine eigens entwickelte Maske des statistischen Softwareprogrammes SPSS. Die Reliabilität der Codierung ist durch Krippendorff's alpha getestet worden (Hayes und Krippendorff 2007). Hierbei ist zu berücksichtigen, dass die Codierung von Tonalität als ,weiche Variable“ eine gewisse Variabiliät zulässt, für die Reliabilitätswerte von 0,60 als akzeptabel gelten (De Swert 2012). In unseren Tests hat sich dieser Wert für allen Tonalitätsvariabeln bei 0,65 eingespielt. Die restlichen Variablen hatten einen Durchschnittswert von 0,84. Die Reliabilität des gesamten Codebuchs liegt damit bei 0,77. Durch Team-Codierung, regelmäßige Absprachen zwischen den Codierern und einer vom Projektleiter durchgeführten Endkontrolle konnte diese Reliabilität weiter verbessert werden.

\section{Der Negativitätsbias in der journalistischen Nachrichtenselektion und im Framing}

\subsection{Nachrichten-Story Negativität}

Die Ergebnisse unserer Studie erlauben es, unmittelbar an die Befunde eines generellen Negativitätsbiases in der Nachrichtenberichterstattung anzuknüpfen (Kepplinger 1998, Soroka 2014). In Deutschland bedienen sich $47 \%$ und in Großbritannien $51 \%$ aller Nachrichten einer diffusen Negativität als Selektionskriterium (gegenüber $8,7 \%$ und 8,6\% positive-gerahmter Nachrichten, siehe Abb. 1). Diese allgemeine Nachrichtennegativität (gute, schlechte oder neutrale Nachrichten) erlaubt allerdings noch keine Schlüsse auf die spezifische Beurteilung der EU. Im Gegenteil kann die außen- oder europapolitische Berichterstattung unter Umständen sogar ausgewogener sein als die politisierten innenpolitischen Nachrichten (siehe hierzu Kepplinger 1998). In der differenzierten Bewertung der EU und ihrer Akteure sind vor allem deutsche Nachrichtenmedien in ihrem Negativurteil zurückhaltender (17,9\% negativer gegenüber 14,5\% positiver Beurteilungen der EU, siehe Abb. 1). Demgegenüber wird in der britischen EU Debatte überwiegend eine ablehnende Haltung gegenüber der EU zum Ausdruck gebracht (35,7\% Negativbewertungen und nur 6,2\% positive Urteile). Differenziert man dieses Bild nach Zeitungen, so fallen vor allem der Telegraph und Daily Mail durch ihre ausschlie $\beta$ lichen Negativ-Beurteilungen der EU ins Auge. Mit anderen Worten, die Journalistinnen und Journalisten dieser Zeitungen 


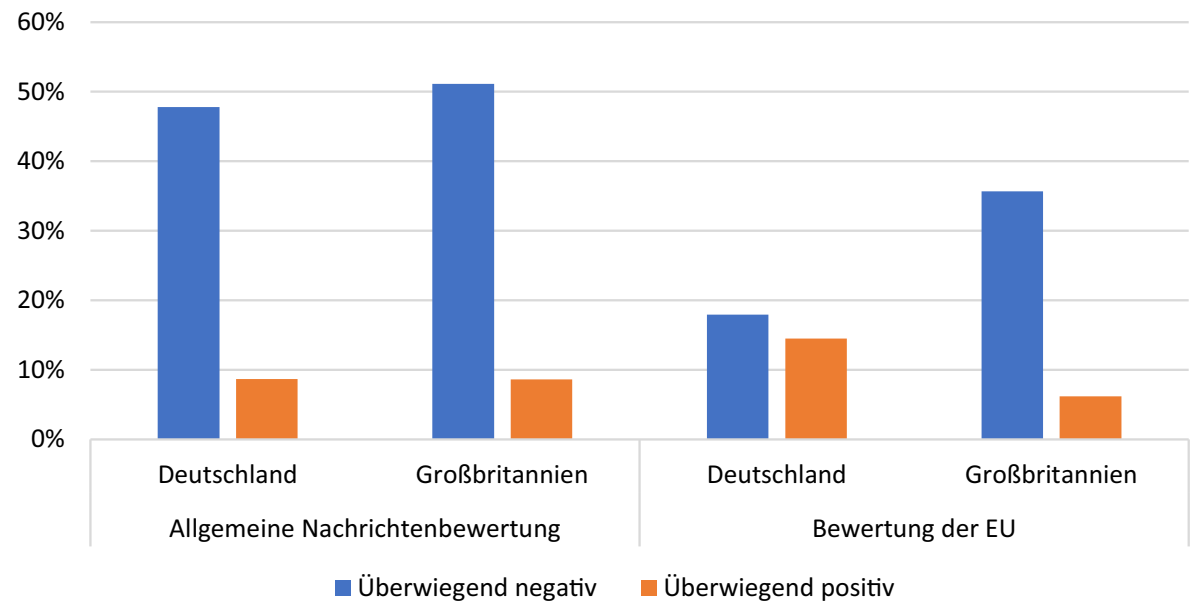

Abb. 1 Nachrichten-Story Negativität

beteiligen sich an einer diffusen euroskeptischen Stimmungsmache und lassen keine vom allgemeinen Negativitätstenor abweichende Meinungen zu.

Die Auswirkungen von Negativität auf die Legitimität der EU unterscheiden sich des Weiteren je nach Inhalten der EU-Kritik, die sich auf politische Entscheidungen der EU beziehen kann (z.B. wenn die Ineffizienz von EU Politik beklagt wird), oder politische Akteure und Institutionen zur Zielscheibe wählen kann (z. B. wenn die Spitzenkandidaten als inkompetent herausgestellt werden) oder den Integrationsprozess grundsätzlich in Frage stellen können. In Anlehnung an David Easton kann Negativität somit Ausdruck des Entzugs von spezifischer oder diffuser Unterstützung sein (Easton 1975). Im ersten Fall bezieht sich die journalistische Negativbeurteilung auf die politischen Entscheidungsprozesse des Regierens (policy), oder auf den parteipolitischen Wahlkampf in der Vergabe von Ämtern der Regierung (politics). Im zweiten Fall bezieht sich die journalistische Negativität auf Beurteilungen von Systemeigenschaften (polity), d.h. sie hinterfragt die Autorität des politischen Systems. ${ }^{6}$ So wie euroskeptische Parteien als Anti-System Parteien bezeichnet werden (De Wilde und Trenz 2012; Risse 2014, S. 15), könnte es also einen AntiSystem Journalismus geben, dem es nicht um die Beurteilung von Input oder Output Legitimation der EU geht, sondern, der den Europäischen Integrationsprozess grundsätzlich in Frage stellt.

Gemä $\beta$ dieser thematischen Einordnung reflektiert die EU-Berichterstattung in Großbritannien einen Mangel an diffuser Unterstützung der EU. Die europäische Parlamentswahl wird vorzugsweise aus der Perspektive nationaler Politik (40,7\%) diskutiert oder es werden grundsätzliche Fragen der Europäischen Integration aufgegriffen (EU-Polity Perspektive 47,1\%) anstatt sich mit den europäischen Institutionen, den Spitzenkandidaten oder auch der Politik der EU im Detail auseinanderzusetzen (Abb. 2). Außerdem fällt die Bewertung der EU-Polity in den meisten Fällen

6 ",Diffuse support' refers to evaluations of what an object is or represents (...) not of what it does" (Easton 1975, S. 444). 


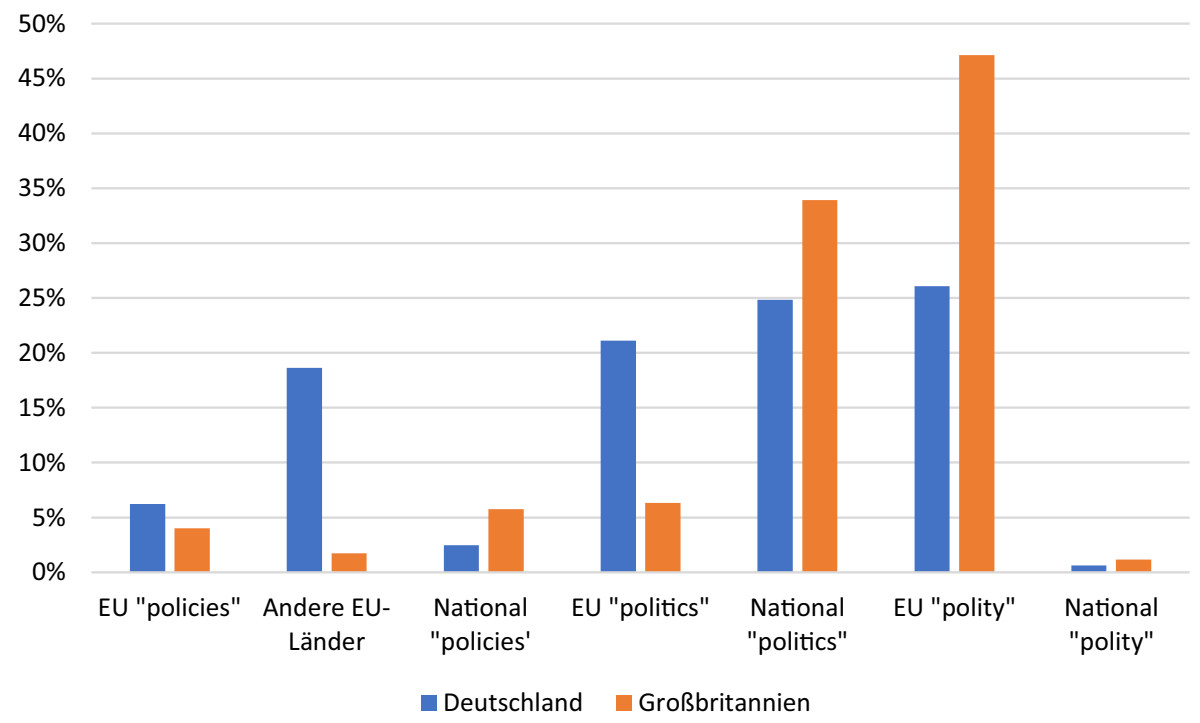

Abb. 2 Thematischer Fokus auf Artikelebene

negativ aus (46,2\% gegenüber 5,1\% positiver Bewertungen - Abb. 3). Ein eigentlicher Wahlkampf fand somit in britischen Medien nicht statt, die Debatte setzte vielmehr ein Vorspiel von Brexit in Szene. Auch in Deutschland findet sich die Tendenz ausgeprägt, die EU im Wahlkampf aus nationaler Perspektive $(27,9 \%)$ oder als Polity $(26,1 \%)$ zu thematisieren und damit die strategische Stoßrichtung der Spitzenkandidatenkampagne zu unterlaufen. Der Umfang dieser Art von second-order Berichterstattung ist allerdings deutlich geringer und zugleich wird der europäischen parteipolitischen Streitpolitik, also den eigentlichen Themen des Wahlkampfs, mehr Aufmerksamkeit geschenkt (21,1\% gegenüber nur 6,3\% in Großbritannien). Auch die Bewertung der EU-Polity ist ausgewogener (23,8\% negativ und 19\% positiv, siehe Abb. 3). Die deutschen Nachrichten berichten auch mehr über den Wahlkampf in anderen Ländern und tragen damit zu einer gesamteuropäischen Perspektive bei (18,6\% gegenüber 1,7\% in Großbritannien, siehe Abb. 2). Dabei handelt es sich vorwiegend (in 56,7\% aller Fälle) um Artikel, die den Euroskeptizismus in anderen Ländern (z.B. Marine le Pen in Frankreich oder Nigel Farage in Großbritannien) zum Thema erheben. Der Euroskeptizismus wird damit nicht als Problem der anderen behandelt, sondern aus europäischer Perspektive thematisiert.

\subsection{Quellen-Negativität}

Auf der Sprecher Ebene folgt die Berichterstattung in beiden Ländern dem traditionellen Muster einer Second-Order Kampagne. Bei den Protagonisten von EUNachrichtenstories handelt es sich in den meisten Fällen um Vertreter nationaler politischer Parteien oder der nationalen Regierung (45,6\% in Deutschland und 60,8\% in Großbritannien). Die Sichtbarkeit von EU Akteuren, wie beispielsweise die Spitzenkandidaten oder die zur Wahl stehenden Mitglieder des Europäischen Parlaments, 


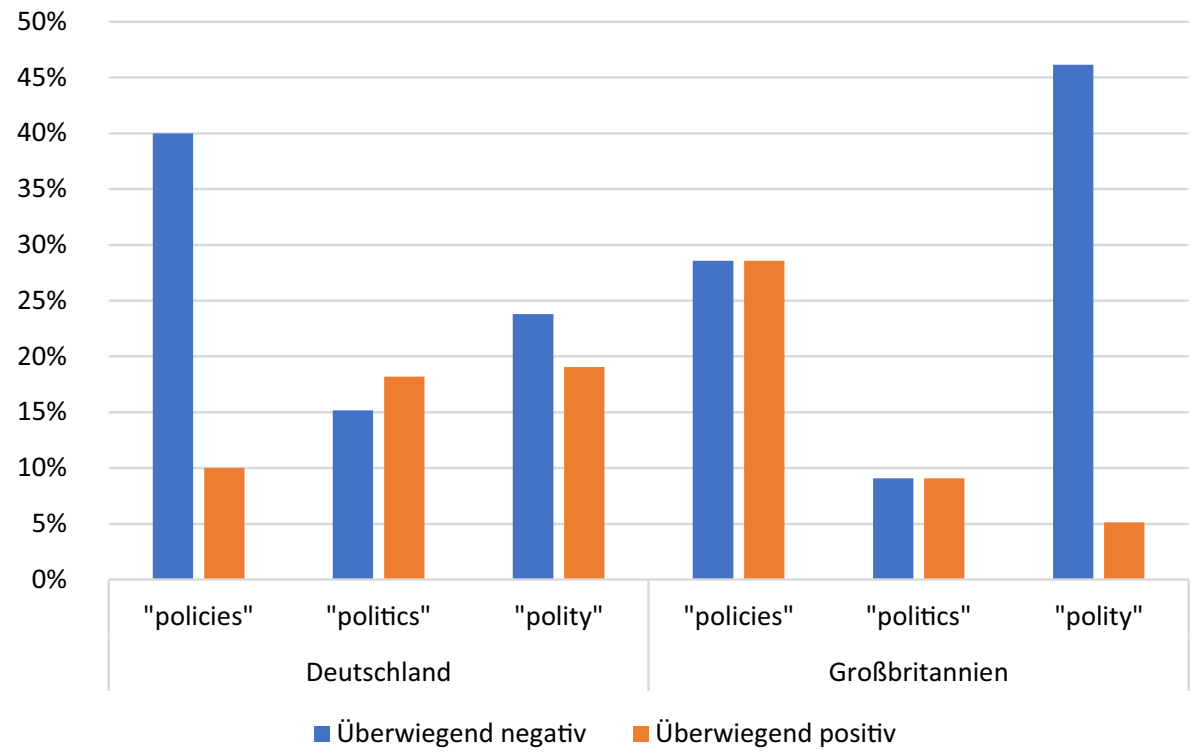

Abb. 3 Bewertung der EU nach thematischem Fokus auf Artikelebene

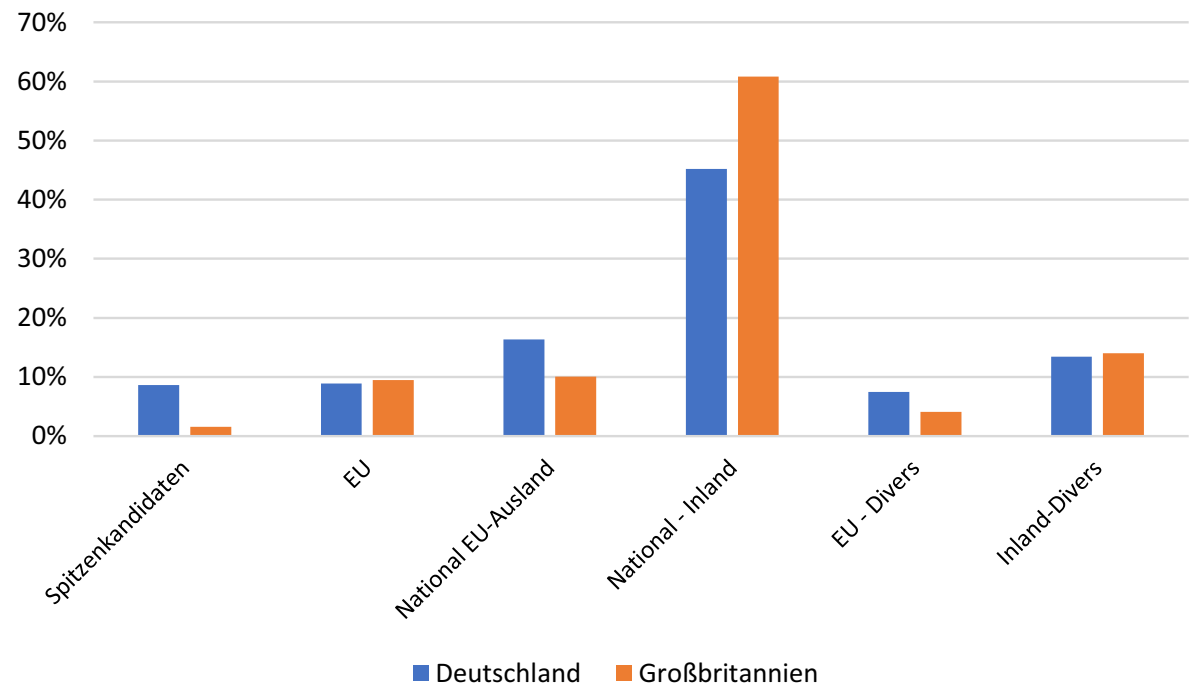

Abb. 4 Sprecher

fällt demgegenüber stark zurück (17,4 in Deutschland und $11 \%$ in Großbritannien, siehe Abb. 4). In der deutschen Presse können immerhin 8,7\% aller Sprechaussagen den Spitzenkandidaten zugeordnet werden. In Großbritannien dagegen bleiben die Kandidaten weitestgehend unsichtbar (1,6\% der Sprechaussagen). Die deutsche Presse zitiert auch deutlich mehr Akteure aus anderen Mitgliedsländern (16,3\% gegenüber $10 \%)$. 


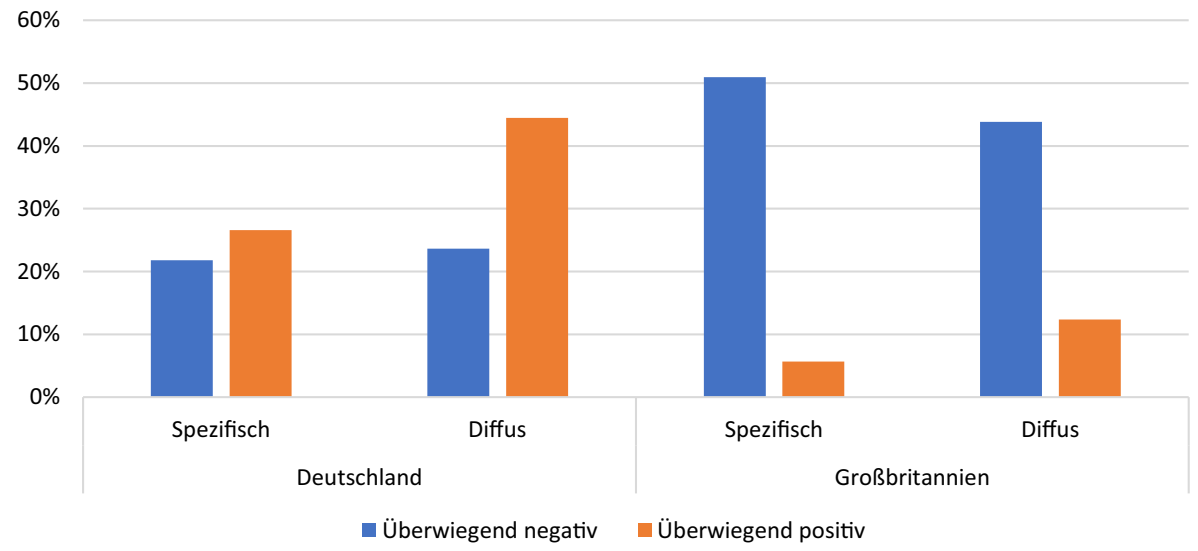

Abb. 5 Spezifische vs. diffuse Sprecherbewertung der EU

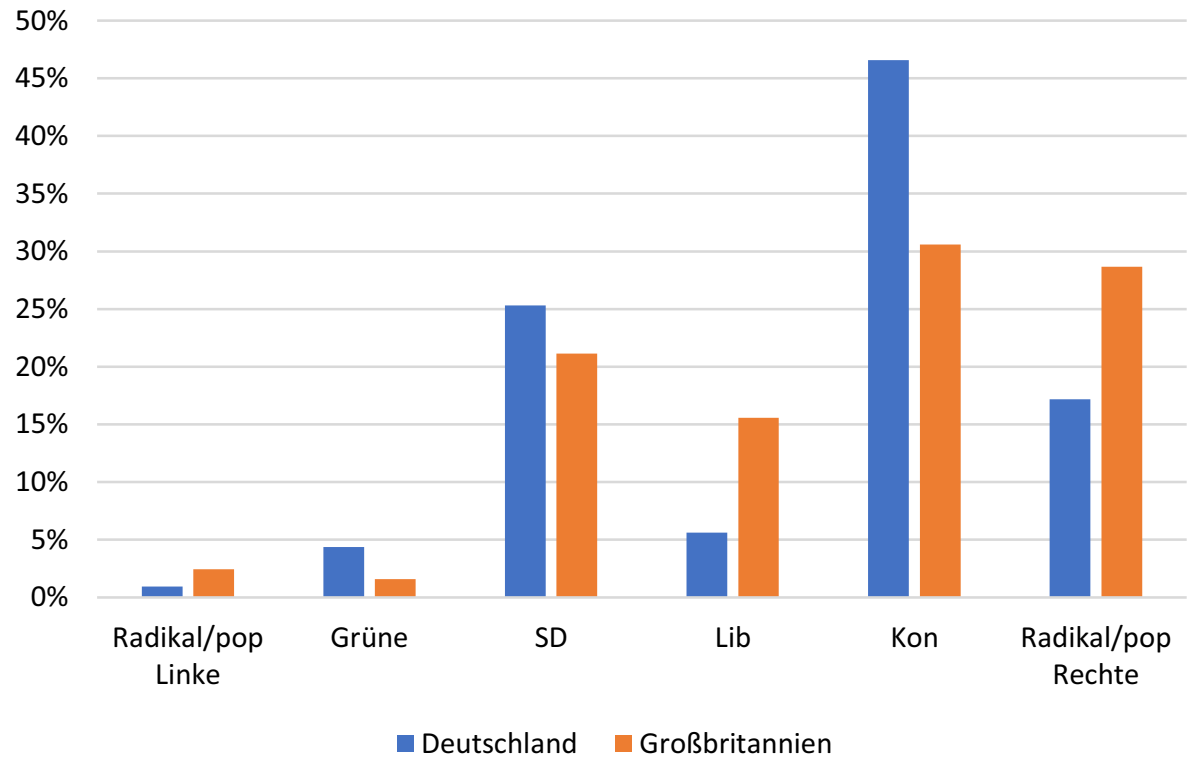

Abb. 6 Parteizughörigkeit von Sprechern

In der Tonalität von zitierten Sprechaussagen bildet die Positivität von Sprechern in der deutschen Berichterstattung einen starken Kontrast zur ausnahmslosen Negativität von Sprechern in den britischen Nachrichtenmedien. Daneben fällt aber vor allem die Diffusität der Europakritik ins Auge, welche den Mangel an diffuser Unterstützung auf Artikelebene sozusagen dupliziert (siehe Abb. 5). In Deutschland überwiegt dabei eine diffuse Positivität in den Sprechaussagen, also eine Art permissiver Konsensus, welcher die EU gutheisst ohne auf spezifische Errungenschaften einzugehen. In Großbritannien hingegen paart sich eine diffuse Negativität mit gezielten Angriffen auf die EU-Institutionen und Akteure. Für beide Länder 
gilt, dass Journalistinnen und Journalisten nicht nur selber im Vagen bleiben, sondern auch mit Vorliebe solche Akteure zitieren, die mit einer Generalkritik der EU hervortreten, ohne sich auf eine ausgewogene Argumentationspraxis einzulassen. Wohingegen deutsche Journalisten die EU-Befürworter als Referenz für ihre Nachrichtenstories bevorziehen, kommen also in der britischen Debatte fast ausschließlich Euroskeptizisten zu Worte. Das hat schwerwiegende Folgen für die Resonanz der Spitzenkandidatenkampagne, die in der britischen Presse ignoriert wird. Britische Journalisten übergehen dabei nicht nur die Kandidaten selber, sie erteilen auch mit Vorliebe der euroskeptischen Opposition das Wort, wodurch die Legitimität der EU weiter unterminiert wird.

In Großbritannien wird der Europawahlkampf in diesem Sinne in der Gestalt einer diffusen, second-order Euroskeptizismus Kampagne ausgefochten. Als besonders erfolgreich im medialen Agenda-Setting erweisen sich dabei Vertreter der radikalen oder populistischen Rechten (28,7\% aller parteipolitischen Sprechaussagen) und konservativer Parteien (mit 30,6\% - siehe Abb. 6) was auch in etwa den Wahlausgang widerspiegelt (26,6\% für UKIP und $23 \%$ für die Conservative Party). Auch in Deutschland werden europaskeptische Positionen rechtsradikaler und rechtspopulistischer Parteien mit medialer Aufmerksamkeit belohnt (17,2\% aller parteipolitischen Sprechaussagen gegenüber 7,1\% Stimmanteil für die AfD im Endergebnis der Wahlen). Insgesamt überwiegen aber mehr ausgewogene second-order parteipolitische Auseinandersetzungen, wobei sich die europapolitische Ebene im Sinne einer first-order Spitzenkandidatenkampagne über ihre prominenten Akteure profilieren kann.

\subsection{Kommentar-Negativität}

In Kontext von Wahlkampagnen kann die Nachrichtenkommentierung als eine Form des politischen Engagements angesehen werden, indem Leserinnen und Leser von der Möglichkeit Gebrauch machen, Meinungen zum politischen Geschehen zu äußern, zur Informationsweitergabe beizutragen und mit anderen Leserinnen und Lesern zum Zwecke des Meinungsaustauschs und der politischen Mobilisierung in Kontakt zu treten (Bossetta et al. 2017; Dahlgren 2013). Die Kommentarspalten von digitalen Nachrichtenportalen werden durch eine lebhafte Community von online Lesern am Leben gehalten, die in kurzen Zeiträumen eine relative hohe Anzahl (oftmals über 500 Kommentare) verfassen. Diese Kommentare erzeugen wiederum Publikumsresonanzen, entweder in der Form direkter Antworten (Zustimmung oder Widerspruch anderer Leser) oder durch ihre Rezeption durch anonyme Zeitungsleser, die es sich zur Gewohnheit machen, nicht nur die journalistischen Nachrichten, sondern auch die Kommentarspalten und damit die Meinungen anderer Leserinnen und Leser zu folgen. Online Leserkommentare sind damit meinungsbildend, aber keineswegs repräsentativ für die die Wahlentscheidungen der Bürgerinnen und Bürger. Sie sind ein fester Bestandteil des online Nachrichtenkonsums, woraus sowohl von den politischen Repräsentanten als auch von anderen Leserinnen und Lesern ein Stimmungsbild zur Wahl abgelesen werden kann. 
Abb. 7 Bewertung der EU in Kommentaren
$100 \%$

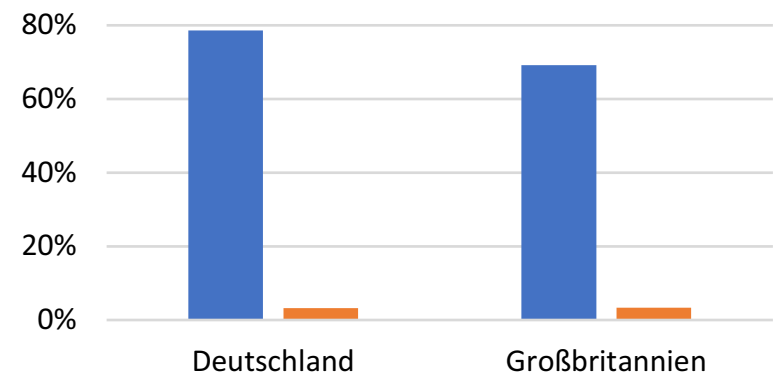

Überwiegend negativ Ü̈berwiegend positiv

Dieses online Stimmungsbild zur Europawahl ist in beiden Ländern dezidiert negativ. In zwei Drittel aller Kommentare zum Thema EU bringen Leserinnen und Leser ihre Abneigung gegenüber der EU zum Ausdruck und nur jeder 25. Leser hat etwas Positives zur EU beizutragen (Abb. 7). Nicht tendenziöse Kommentare finden sich in etwa jedem dritten Beitrag. Dabei kann es sich um ausgewogene Stellungnahmen zum politischen Geschehen oder Beiträgen zur Informationsgewinnung handeln, in dem meisten Fällen aber war aufgrund der Uneindeutigkeit oder Dekontextualisierung der Sprechaussage eine Zuordnung von Tonalität nicht möglich.

In den Leserkommentaren nivellieren sich auch die auf Nachrichten- und Sprecherebene gefundenen Unterschiede zwischen Deutschland und Großbritannien. Journalisten oder Sprecher, die sich in Deutschland überwiegend positiv zu EU zu Wort melden, finden also auf der Rezipientenebene keine Resonanz bzw. müssen mit negativen Publikumsreaktionen rechnen.

Gemäß der Hypothese, dass Negativität Leser kritisch engagiert, können wir ferner die Erwartung formulieren, dass negativ gerahmte Nachrichten einen höheren Grad an Leserengagement fördern. Das trifft auf beide Länder zu (siehe Tab. 1). Negative Nachrichtenstories können die Leserinnen und Leser aufmuntern, von der Kommentarfunktion Gebrauch zu machen und sich in europapolitischen Debatten kritisch zu Wort zu melden. Eine solche Korrelation findet sich in beiden Ländern nur schwach ausgeprägt. In Antwort auf positiv gerahmte Artikel ist die Anzahl von Negativkommentaren zwar geringfügig niedriger $(66 \%$ gegenüber $75 \%$ negativer Kommentare). Darüber hinaus aber ist die Stimmung in den Kommentarspalten der Zeitungen unabhängig von der Tonalität des Hauptnachrichtenartikels unisono negativ. Wer sich positiv zur EU äußern möchte, ortet sich klar als Außenseiter ( $1,1 \%$ aller Leserkommentare in Deutschland und 2,4\% in Großbritannien).

Länderspezifika in der Leserkommentierung zur EU lassen sich hingegen hinsichtlich der Polity-Beurteilung der EU bzw. der Beurteilung von EU-Politik oder ihrer politischen Repräsentanten ausmachen (Tab. 2). Deutsche Leser zeigen eher eine Bereitschaft, zwischen diesen Ebenen zu differenzieren und platzieren ihre negativen Kommentare sowohl als Beitrag zu sogenannter EU-Polity-Debatten, als auch als Beitrag zur Spitzenkandidaten-Debatten. Ein solcher differenzierterer Blick auf Europa kommt in der britischen Presse nicht zur Anwendung, zum einen 
Tab. 1 Bewertung der EU in Kommentaren und Artikeln (neutral/ambivalent nicht ausgewiesen)

\begin{tabular}{llllll}
\hline & $\begin{array}{l}\text { Deutschland } \\
\text { Artikel } \\
\text { überwiegend } \\
\text { negativ }\end{array}$ & $\begin{array}{l}\text { Artikel } \\
\text { überwie- } \\
\text { gend positiv }\end{array}$ & $\begin{array}{l}\text { Artikel überwie- } \\
\text { gend negativ }\end{array}$ & $\begin{array}{l}\text { Artikel } \\
\text { überwie- } \\
\text { gend positiv }\end{array}$ \\
\hline Kommentar überwiegend negativ & $75,0 \%(66)$ & $66,7 \%(16)$ & $71,9 \%(105)$ & $51,9 \%(14)$ & 201 \\
Kommentar überwiegend positiv & $1,1 \%(1)$ & $4,2 \%(1)$ & $2,1 \%(3)$ & $11,1 \%(3)$ & 8 \\
\hline
\end{tabular}

Tab. 2 Bewertung der EU in Kommentaren nach thematischem Fokus auf Artikelebene (neutral/ ambivalent nicht ausgewiesen)

\begin{tabular}{|c|c|c|c|c|c|c|c|}
\hline & $\begin{array}{l}\text { EU ,po- } \\
\text { licies““ }\end{array}$ & $\begin{array}{l}\text { Andere } \\
\text { EU- } \\
\text { Länder }\end{array}$ & $\begin{array}{l}\text { National } \\
\text { „poli- } \\
\text { cies“ }\end{array}$ & $\begin{array}{l}\text { EU } \\
\text { „politics“ }\end{array}$ & $\begin{array}{l}\text { National } \\
\text { „politics“ }\end{array}$ & $\begin{array}{l}\text { EU ,poli- } \\
\text { ty“" }\end{array}$ & Total \\
\hline \multicolumn{8}{|l|}{ Deutschland } \\
\hline $\begin{array}{l}\text { Überwiegend } \\
\text { negativ }\end{array}$ & $\begin{array}{l}4,9 \% \\
(13)\end{array}$ & $\begin{array}{l}19,2 \% \\
(51)\end{array}$ & $1,5 \%(4)$ & $\begin{array}{l}29,1 \% \\
(77)\end{array}$ & $\begin{array}{l}12,8 \% \\
(34)\end{array}$ & $\begin{array}{l}32,5 \% \\
(86)\end{array}$ & 265 \\
\hline $\begin{array}{l}\text { Überwiegend } \\
\text { positiv }\end{array}$ & $0,0 \%(0)$ & $18,2 \%(2)$ & $0,0 \%(0)$ & $45,5 \%(5)$ & $9,1 \%(1)$ & $27,3 \%(3)$ & 11 \\
\hline \multicolumn{8}{|l|}{ Großbritannien } \\
\hline $\begin{array}{l}\text { Überwiegend } \\
\text { negativ }\end{array}$ & $4,2 \%$ (7) & $1,8 \%$ (3) & $\begin{array}{l}6,0 \% \\
(10)\end{array}$ & $3,0 \%(5)$ & $\begin{array}{l}12,7 \% \\
(21)\end{array}$ & $\begin{array}{l}72,3 \% \\
(120)\end{array}$ & 166 \\
\hline $\begin{array}{l}\text { Überwiegend } \\
\text { positiv }\end{array}$ & $0,0 \%(0)$ & $0,0 \%(0)$ & $0,0 \%(0)$ & $0,0 \%(0)$ & $12,5 \%(1)$ & $87,5 \%(7)$ & 8 \\
\hline
\end{tabular}

weil die Berichterstattung selber keine Differenzierung zulässt, zum anderen weil die Leserkommentierung fast ausschliesslich darauf abzielt, die EU-Mitgliedschaft Großbritanniens fundamental in Frage zu stellen. Wie die journalistische Berichterstattung, orientieren sich auch die Leserinnen und Leser in Großbritannien bereits an der Leitvorstellung eines Austritts aus der EU (Brexit).

\section{Schlussbetrachtung: Nachrichten-Negativität und demokratische Kontrolle in der EU}

Abschließend soll die Frage nach der Wirkung von Nachrichten-Negativiät in Hinblick auf die demokratische Kontrollfunktion des Journalismus im Zusammenhang der Europawahlen aufgeworfen werden Im Zusammenhang mit den kritischen Funktionen des Journalismus kann Mediennegativität als Gradmesser jeder gesunden Demokratie angesehen werden, in der Journalisten Distanz zum politischen Geschehen halten, ihre Leser informieren und einladen, sich kritisch mit der Politik auseinanderzusetzen (Soroka 2014). In westlichen Demokratien ist in diesem Sinne der Anstieg von Negativnachrichten in den Sechziger und Siebziger Jahren mit dem Heranwachsen einer neuen Generation kritischer Journalisten in Zusammenhang gebracht werden (Kepplinger 1998, S. 144). Negativität in der Nachrichtenberichterstattung korreliert mit dem Wunsch des Journalismus, exisitierende Machstrukturen herauszufordern und Politiker zur Verantwortung zu ziehen. 
Die Negativität in der EU-Nachrichtenberichterstattung könnte also von der Motivation der Journalistinnen und Journalisten angetrieben werden, die Wahlen zum Europäischen Parlament als Gelegenheitsstruktur zur Herstellung kritischer Öffentlichkeit zu nutzen. Ohne eine solche Motivation des Journalismus in Abrede zu stellen, fällt die EU-Berichterstattung jedoch durch eine Reihe von Besonderheiten auf, welche die Funktion kritischer Öffentlichkeit unterlaufen. Hier wäre vor allem auf die Art und Weise der medial vermittelten politischen Konfrontation hinzuweisen, durch die eine fundamentale Systemkritik der EU in den Vordergrund, parteipolitische Auseinandersetzungen hingegen in den Hintergrund gestellt werden. Mediennegativität wird also nicht in Kritik mit der Programmatik des europäischen Regierens übersetzt, sondern bleibt diffus. Eine solche diffuse Negativität, wie sie insbesondere in der britischen Presse herausgestellt wird, kann nicht als Hebel politischer Kontrolle eingesetzt werden. Sie wirkt allenfalls demotivierend auf die Entscheidungen der Wählerinnen und Wähler, sich am demokratischen Entscheidungsprozess zu beteiligen und verstärkt die Tendenz, der EU ihre Basislegitimation abzusprechen, statt, wie es von der Wahl gefordert wird, sich mit der Frage nach der Qualität ihres politischen Programmes auseinanderzusetzen.

Auf kognitiver Ebene der Nachrichtenrezeption ist ferner behauptet worden, dass negativ gerahmte Nachrichtenstories einen höheren Informationswert besitzen und die Problemwahrnehmungsfähigkeit der Leserinnen und Leser generell stärken können (Dunaway et al. 2015, S. 783; Scheufele 2008). Hier können wir durch unsere Lesermeinungsanalyse einen direkten Wirkungszusammenhang zwischen Nachrichtenrahmung und Rezeption herstellen. Die Befunde weisen dabei eher auf ein verzerrtes Meinungsbild zur EU, das durch diffuse Negativität genährt wird. Die Leserinnen und Leser sind nicht in einen politischen Streit eingebunden, sondern üben sich in der Diffamierung ihrer politischen Repräsentanten, wobei insbesondere die Integrität von EU-Akteuren radikal in Abrede gestellt wird. Sie treiben somit die Negativität mit der EU auf die Spitze und verfestigen ein einseitiges euroskeptisches Meinungsbild.

Die Erwartung, die Spitzenkandidatenkampagne für eine informierte Meinungsbildung der Wählerinnen und Wähler zum politischen Geschehen der EU einsetzen zu können, wird dementsprechend von den Nachrichtenmedien nur bedingt eingelöst. Die in dieser Studie zugrundegelegte These einer institutionellen Eigenlogik der Nachrichtenmedien ist hilfreich, um das begrenzte Demokratisierungspotential europäischer Wahlen herauszuarbeiten. In Ermangelung eines europäischen Journalismus müssen sich die Repräsentanten europäischer Demokratie nach wie vor auf die Dienstleistungen nationaler Medienorganisationen verlassen. Die Europaberichterstattung unterliegt dabei einer Reihe von nationalstaatlich spezifischer Selektionskriterien, wobei die journalistischen Qualitätsstandards der neutralen Nachrichtenübermittlung und der am Gemeinwohl orientierten Kritik nur begrenzt zum Einsatz gelangen. In der vorliegenden Studie konnte insbesondere die Negativität als systematischer Medienbias in der Europaberichterstattung herausgearbeitet werden. Im Falle der Negativität unterscheidet sich der journalistische Stil von dem des Informationsvermittlers und des Kritikers dadurch, dass Nachrichten schablonenhaft, aber dennoch effizient und den Erwartungshaltungen des Publikums entsprechend hergestellt werden können (Bourdieu 1998). Negative Nachrichten sind 
schnelle Nachrichten, wohingegen kritische Reflektion zeitaufwendig ist und auch Medienraum beansprucht, also Kapazitäten wie kostbare Nachrichtenspalten oder langwierige Recherchen (ibid., S. 39), die in der Europaberichterstattung zumeist nicht zur Verfügung stehen.

Als Wirkungszusammenhang von Nachrichtenberichterstattung und politischer Meinungsbildung zur EU bietet sich die bereits viefach ausgetestete These einer Negativitätsspirale an, die hier im Kontext der online Nachrichtenrezeption neue Relevanz gewinnt (De Vreese 2007; De Wilde et al. 2013). Hier wäre zunächst auf eine mögliche Korrelation zwischen Mediennegativität und politischem Desinteresse und Desinformation (siehe hierzu auch Patterson 2000, S. 10) hinzuweisen. Zur Analyse des Rezeptionsverhaltens von Nachrichtenkonsumenten kann die hier vorliegende Inhaltsanalyse von Nachrichtenselektion und -rahmung allerdings nur bedingt beitragen. Durch die im Online Angebot von Nachrichten unterbreitete Möglichkeit der Kommentierung erhalten wir allenfalls einen nicht-repräsentativen Einblick in das Stimmungsbild der Wählerschaft. Das online Rezeptionsverhalten liefert jedoch einen eindrucksvollen Beweis der Relevanz der These einer Negativitätsspirale im Zusammenwirken von EU-Nachrichtenberichterstattung und politischer Meinungsbildung. Berücksichtigt man weiterhin, dass diese Art der Kommentierung auf vielbesuchten Nachrichtenseiten von einflussreichen Zeitungen erfolgt, muss von einer entsprechenden Resonanz auch bei unbeteiligten Lesern ausgegangen werden. Die These eines ,vicious circle ' der in der institutionellen Medienlogik inhärenten Nachrichtennegativität findet sich somit bestätigt: wer sich überwiegend mit Negativschlagzeilen konfrontiert sieht, verliert sein Vertrauen in die Politik und entwickelt gleichzeitig entsprechende Erwartungshaltungen in die Negativität von Nachrichten. Parallel hierzu werden Journalistinnen und Journalisten durch Marktlogiken bestärkt, sich mit negativen Nachrichten zu profilieren, um den Erwartungshaltungen des Publikums entgegenzukommen. Diese Negativitätsspirale dreht sich in der EU-Berichterstattung noch schneller, da die Medien dem politischen Geschehen in der EU ohnehin nur eine geringe Aufmerksamkeit schenken. Bei verminderter Qualität des Informationsangebots zur EU-Politik bleiben Negativnachrichten zumeist unwidersprochen und können das Vertrauen in die EU-Demokratie ungebremst unterminieren (Clark 2014).

Daneben stellen sich die Rezeptionsbedingungen für die Spitzenkandidatenkampagne sehr unterschiedlich dar, wodurch der europäische Kommunikationsraum zerfällt und keiner einheitlichen politischen Willensbildung zugeführt werden kann. Dies konnte in der vorliegenden Studie an den beiden Fällen Deutschland und Großbritannien exemplarisch dargelegt werden. In Anbetracht der fragmentierten Medienlandschaften des politischen Europas muss deshalb weiterhin mit unterschiedlichen Produktions-, Distributions- und Rezeptionskontexten von Nachrichten gerechnet werden, die für ein differenziertes Politisierungsmuster der EU verantwortlich zeichnen (de Wilde et al. 2016). Die Schaffung von politisch-institutionellen Gelegenheitsstrukturen für eine demokratische Autorisierung des Repräsentationsgefüges der EU, wie hier in der Form der Spitzenkampagne untersucht, bleibt für die Herstellung einer legitimierenden Öffentlichkeit unzureichend. 
Open Access Dieser Artikel wird unter der Creative Commons Namensnennung 4.0 International Lizenz (http://creativecommons.org/licenses/by/4.0/deed.de) veröffentlicht, welche die Nutzung, Vervielfältigung, Bearbeitung, Verbreitung und Wiedergabe in jeglichem Medium und Format erlaubt, sofern Sie den/die ursprünglichen Autor(en) und die Quelle ordnungsgemäß nennen, einen Link zur Creative Commons Lizenz beifügen und angeben, ob Änderungen vorgenommen wurden.

\section{Literatur}

Adam, Silke. 2009. Euroscepticism and the mass meda. An analysis of the form of contention in the German and French debates on a European constitution. In Euroscepticism. Images of Europe among mass publics and political elites, Hrsg. D. Fuchs, R. Magni-Berton, und A. Roger, 193-211. Opladen: Barbara Budrich.

Altheide, David L., und Robert P. Snow. 1979. Media logic. Beverly Hills, London: SAGE.

Arditi, Benjaim. 2007. Politics on the edges of liberalism: difference, populism, revolution, agitation. Edinburgh: Edinburgh University Press.

Bach, Maurizio. 2008. Europa ohne Gesellschaft: Politische Soziologie der Europäischen Integration. Wiesbaden: VS.

Bossetta, Michael, Anamaria Dutceac Segesten, und Hans-Jörg Trenz. 2017. Transnational citizen engagement through social networking media: the factual, the ideological, and the moral style. In Social media and European politics, Hrsg. Mauro Barisone, Asimina Michailidou. Basingstoke: Palgrave.

Bourdieu, Pierre. 1998. Über das Fernsehen. Frankfurt a.M: Suhrkamp.

Brüggemann, Michael. 2010. Information policy and the public sphere: EU communications and the promises of dialogue and transparency. Javnost-The Public 17(1):5-22.

van Brussel, Annelies. 2014. From informing to interacting? Exploring the European commission's communication strategy "to be all ears". Journal of Contemporary European Research 10(1):90-104.

Cappella, James N., und Kathleen Jamieson. 1997. Spiral of cynicism: the press and the public good. Oxford: Oxford University Press.

Clark, Nicholas. 2014. The EU's information deficit: comparing political knowledge across levels of governance. Perspectives on European Politics and Society 15(4):445-463.

Daddow, Oliver. 2012. The UK media and 'Europe': from permissive consensus to destructive dissent. International Affairs 88(6):1219-1236.

Dahlgren, Peter. 2013. The political web. Media, participation and alternative democracy. Basingstoke: Palgrave Macmillan.

Diez Medrano, Juan. 2003. Framing europe: attitudes to European integration in Germany, Spain, and the United Kingdom. Princeton: Princeton University Press.

DiMaggio, Paul J., und William W. Powell. 1983. The iron cage revisited-institutional isomorphism and collective rationality in organizational fields. American Sociological Review 48(2):147-160.

Dunaway, Johanna L., T. Davis Nicholas, Jeremy Padgett, und Rosanne M. Scholl. 2015. Objectivity and information bias in campaign news. Journal of Communication 65(5):770-792.

Easton, David. 1975. A re-assessment of the concept of political support. British Journal of Political Science 5(04):435-457.

Entman, Robert M. 2004. Projections of power. Framing news, public opinion and US foreign policy. Chicago: University of Chicago Press.

Galtung, Johan, und Marie H. Ruge. 1965. The structure of foreign news. Journal of Peace Research 2(1):64-91.

Gattermann, Katja, und Sofia Vasilopoulou. 2017. Eurosceptic candidate MEPs in the news: a transnational perspective. In Euroscepticism as a transnational and pan-European phenomenon: the emergence of a new sphere of opposition, Hrsg. J. Fitz Gibbon, B. Leruth, und N. Startin, 130. London: Routledge.

Gattermann, Katja, Claes H. Vreese, und Wouter Brug. 2016. Evaluations of the Spitzenkandidaten: the role of information and news exposure in citizens' preference formation. Politics and Governance 4(1):37-54.

Gerhards, Jürgen, Silke Hans, und Sören Carlson. 2016. Klassenlage und transnationales Humankapital: Wie Eltern der mittleren und oberen Klassen ihre Kinder auf die Globalisierung vorbereiten. Wiesbaden: Springer VS.

Hanitzsch, Thomas. 2016. Das journalistische Feld. In Handbuch Journalismustheorien, Hrsg. M. Löffelholz, L. Rothenberger, 281-293. Wiesbaden: Springer VS.

Hannerz, Ulf. 2004. Foreign news. Exploring the world of foreign correspondents. Chicago: University of Chicago Press. 
Hayes, Andrew F., und Klaus Krippendorff. 2007. Answering the call for a standard reliability measure for coding data. Communication Methods and Measures 1:77-89.

Hepp, Andreas, Michael Brüggemann, Katharina K. Königslöw, Swantje Lingenberg, und Johanna Möller. 2012. Politische Diskurskulturen in Europa: Die Mehrfachsegmentierung europäischer Öffentlichkeit. Wiesbaden: VS.

Hix, Simon, und Michael Marsh. 2007. Punishment or protest? Understanding European parliament elections. The Journal of Politics 69(2):495-510.

Hjarvard, Stig. 2013. The mediatization of culture and society. London: Routledge.

Hooghe, Lisbeth. 2007. Understanding Euroscepticism. Acta Politica 42(2/3):231.

Hooghe, Lisbeth, und Gerry Marks. 2009. A Postfunctionalist theory of European integration: from permissive consensus to constraining dissensus. British Journal of Political Science 39(1):1-23.

Hummel, Roman. 2006. Journalistisches Feld und europäische Öffentlichkeit. In Europäische Öffentlichkeit und medialer Wandel, Hrsg. W.R. Langenbucher, M. Latzer. Wiesbaden: VS.

Kepplinger, Hans. 1998. Die Demontage der Politik in der Informationsgesellschaft. Freiburg, München: Alber.

Kiess, Johannes, Elmar Brähler, Gabriele Schmutzer, und Oliver Decker. 2017. Euroscepticism and rightwing extremist attitudes in Germany: a result of the 'dialectic nature of progress'? German Politics 26(2):235-254.

Koopmans, Ruud, und Paul Statham. 2010. Theoretical framework, research design, and methods. In The making of a European public sphere. Media discourse and political contention, Hrsg. Ruud Koopmans, Paul Statham, 34-59. Cambridge: Cambridge University Press.

Kriesi, Hanspeter, Edgar Grande, Martin Dolezal, Marc Helbling, Dominik Höglinger, Sven Hutter, und Bruno Wüst. 2012. Political conflict in Western Europe. Cambridge: Cambridge University Press.

Lengauer, Günther, Frank Esser, und Rosa Berganza. 2012. Negativity in political news: a review of concepts, operationalizations and key findings. Journalism 13(2):179-202.

Machill, Marcel, Markus Beiler, und Corinna Fischer. 2006. Europa-Themen in Europas Medien - die Debatte urn die europäische Öffentlichkeit. In Europäische Öffentlichkeit und medialer Wandel: Eine transdisziplinäre Perspektive, Hrsg. Wolfgang R. Langenbucher, Michael Latzer, 132-155. Wiesbaden: VS.

Marcinkowski, Frank. 2005. Die „Medialiserbarkeit“ politischer Institutionen. In The media society and its myths, Hrsg. P. Rössler, F. Krotz. Konstanz: UVK.

Marcinkowski, Frank, und Adrian Steiner. 2009. Was heisst Medialisierung. Autonomiebeschraenkung oder Ermoeglichung von Politik durch Massenmedien, National Centre of Competence in Research (NCCR) Challenges to Democracy in the 21st Century Working Paper No. 2. http://www.nccrdemocracy.uzh.ch/publications/workingpaper/pdf/WP29.pdf. Zugegriffen: 04.03.2018

Nassehi, Armin. 2002. Politik der Staates oder Politik der Gesellschaft. Kollektivität als Problemformel der Politischen. In Niklas Luhmanns politische Soziologie, Hrsg. Kai-Uwe Hellemann, Rainer SchmalzBruns, 38-59. Frankfurt a. M: Suhrkamp.

Nielsen, Julie H., und Mark N. Franklin. 2016. The Eurosceptic 2014 European parliament elections: second order or second rate? Basingstoke: Palgrave Macmillan.

Örnebring, Hendrik. 2013. Questioning European journalism. Journalism Studies 10(1):2-17.

Patterson, Thomas E. 2000. Doing well and doing good: how soft news and critical journalism are shrinking the news audience and weakening democracy — and what news outlets Can do about it. John F. Kennedy School of Government Faculty Research Working Papers Series, RWP01-001. Cambridge: Harvard University.

Reif, Karl Heinz, und Hermann Schmitt. 1980. Nine second-order national elections-a conceptual framework for the analysis of European election results. European Journal of Political Research 8(1):3-44.

Risse, Thomas (Hrsg.). 2014. European public spheres. Cambridge: Cambridge University Press.

Scheufele, Bernd. 2008. Die These der Negativitätsumkehrung: Ein Experiment zur Wirkung von Negativität in den Politiker- und Problemdarstellungen von Printmedien. Publizistik 53(1):48-64.

Schmidt, Vivian A. 2008. Discursive institutionalism: the explanatory power of ideas and discourse. Annual Review of Political Science 11(1):303-326.

Schmitt, Hermann. 2005. The European parliament elections of June 2004: still second-order? West European Politics 28(3):650-679.

Soroka, Stuart N. 2014. Negativity in democratic politics: causes and consequences. Cambridge: Cambridge University Press.

De Swert, Knut. 2012. Calculating inter-coder reliability in media content analysis using Krippendorff's Alpha. Amsterdam: Center for Politics and Communication, University of Amsterdam. 
Taggart, Paul, und Aleks Szczerbiak. 2004. Contemporary Euroscepticism in the party systems of the European Union candidate states of Central and Eastern Europe. European Journal of Political Research 43(1):1-27.

Teney, Céline, Onawa P. Lacewell, und Pieter De Wilde. 2013. Winners and losers of globalization in Europe: attitudes and ideologies. European Political Science Review 6(4):575-595.

Trenz, Hans-Jörg. 2005a. Die mediale Odrnung der politischen Europas: Formen und Dynamiken der Europäisierung politischer Kommunikation der Qualitätspresse. Zeitschrift für Soziologie 34(3):198-215.

Trenz, Hans-Jörg. 2005b. Europa in den Medien. Die europäische Integration im Spiegel nationaler Öffentlichkeit. Frankfurt, New York: Campus.

Trenz, Hans-Jörg. 2008. Understanding media impact on European integration: enhancing or restricting the scope of legitimacy of the EU? Journal of European Integration 30(2):291-309.

De Vreese, Claes H. 2003. Framing Europe: television news and European integration. Amsterdam: Askant.

De Vreese, Claes H. 2007. A spiral of Euroscepticism: the media's fault? Acta Politica 42(2/3):271-286.

De Vreese, Claes H., und Anna Kandyla. 2009. News framing and public support for a common foreign and security policy. Journal of Common Market Studies 47(3):453-481.

De Vries, Catherine E., und Erica E. Edwards. 2009. Taking Europe to its extremes: extremist parties and public Euroscepticism. Party Politics 15(1):5-28.

De Wilde, Pieter, und Hans-Jörg Trenz. 2012. Denouncing European integration: Euroscepticism as polity contestation. European Journal of Social Theory 15(4):537-554.

De Wilde, Pieter, Aasimina Michailidou, und Hans-Jörg Trenz. 2013. Contesting Europe. Exploring Euroscepticism in online media coverage. Colchester: ECPR Press.

de Wilde, Pieter, Anna Leupold, und Henning Schmidtke. 2016. Introduction: the differentiated politicisation of European governance. West European Politics 39(1):3-22.

Charlotte Galpin ist Dozentin in deutscher und europäischer Politik und stellvertretende Direktorin des Instituts für Deutschlandstudien an der Universität Birmingham, Großbritannien.

Hans-Jörg Trenz ist Professor am Centre for Modern European Studies der Universität Kopenhagen und Forschungsprofessor bei ARENA, Centre for European Studies der Universität Oslo. 